\title{
Study warns of burgeoning health burden of CKD in China
}

A

$\mathrm{n}$ estimated 119.5 million individuals in China are affected by chronic kidney disease (CKD), according to a national survey. The survey's findings, which are published in The Lancet, reveal that CKD has become an important public health problem in China, particularly in economically developing rural areas, and is likely to have substantial socioeconomic and public health consequences.

CKD is known to be prevalent in developing countries; however, nationwide data on CKD prevalence in developing countries that take into account ethnic and economic diversity are sparse. To obtain a reliable estimate of the prevalence of CKD in China and explore factors associated with CKD, Luxia Zhang and colleagues performed a cross-sectional study of a representative sample of adults in the general Chinese population. "A major strength of our study is that it ... provides a unique dataset with which to explore the effect of multiple demographic variables on the burden and trends of chronic kidney disease", they state.

The researchers used a randomized, multistage, stratified sampling approach to obtain their study population. Of 50,550 individuals invited to participate, 47,204 agreed. The researchers asked participants to complete a questionnaire to assess socioeconomic status, health history and lifestyle, took blood pressure measurements, and obtained blood and urine samples. Urinary albumin and creatinine levels were used to assess albuminuria and serum creatinine levels were used to determine estimated glomerular filtration rate (eGFR) using an adapted version of the Modification of Diet in Renal Disease equation. CKD was defined as eGFR $<60 \mathrm{ml} / \mathrm{min} / 1.73 \mathrm{~m}^{2}$ or the presence of albuminuria.

The adjusted prevalence of eGFR $<60 \mathrm{ml} / \mathrm{min} / 1.73 \mathrm{~m}^{2}$ among participants was $1.7 \%$ (95\% CI $1.5-1.9 \%)$ whereas the adjusted prevalence of albuminuria was 9.4\% (95\% CI 8.9-10.0\%). The overall prevalence of CKD was $10.8 \%$ (95\% CI

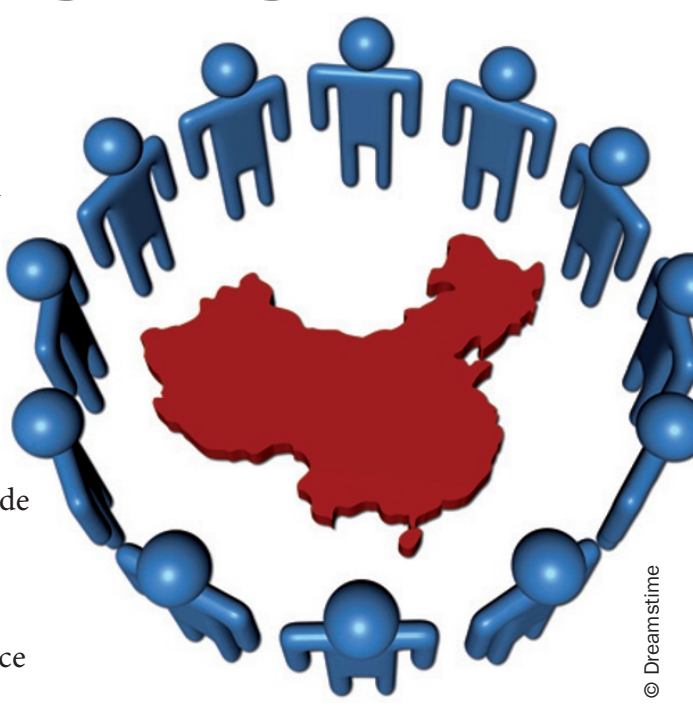

diabetes were independently associated with albuminuria.

To investigate other possible factors associated with CKD, Zhang et al. stratified CKD prevalence by economic development as assessed by tertiles of gross domestic product (GDP) per head. They identified a positive association between the presence of albuminuria and economic development in rural areas, such that the prevalence of albuminuria was highest in the third tertile of GDP per head. The researchers suggest that this finding could either be caused by a disparity in health care between urban and rural areas or by factors not captured by their study, such as an unhealthy lifestyle associated with increased economic development and low health literacy.

Zhang et al. also investigated researchers, equates to approximately 119.5 million individuals across China. As pointed out in an accompanying commentary by Csaba Kovesdy and Kamyar Kalantar-Zadeh, this number dwarfs the estimated 26.3 million people with CKD in the USA.

Zhang and colleagues found that the prevalence of albuminuria was higher in rural than in urban areas $(10.1 \%$ versus $7.0 \%$ ) whereas the prevalence of eGFR $<60 \mathrm{ml} / \mathrm{min} / 1.73 \mathrm{~m}^{2}$ was higher in urban than in rural areas (2.3\% versus $1.6 \%)$. Patients in rural areas had lower levels of education, poorer control of hypertension and a reduced awareness of diabetes than patients from urban areas. Kovesdy and Kalantar-Zadeh warn that if correct, these results could cause a substantial public health problem for China: “...in the near future a large number of patients might need renal replacement therapy in areas where the current healthcare system is ill-equipped to provide such care", they state.

Only $12.5 \%$ (95\% CI 10.6-14.4\%) of participants were aware of CKD. Age, female sex, history of cardiovascular disease, diabetes and hyperuricemia were all independently associated with having an $\mathrm{eGFR}<60 \mathrm{ml} / \mathrm{min} / 1.73 \mathrm{~m}^{2}$ whereas age, female sex, hypertension and geographical variations in CKD prevalence within China. They found a greater prevalence of CKD in the north and southwest of China than in other regions, which they suggest could be explained by unmeasured genetic and environmental confounders.

Although the estimated prevalence of CKD in this study is comparable with estimates for developed countries, such as the USA and Norway, the researchers found that the prevalence of stage 3 and stage 4 CKD was much lower in China than in developed countries. They propose that this difference might be because the development of CKD lags behind increases in hypertension and diabetes. However, they also believe that such a lag provides a unique opportunity for intervention, with special attention given to residents in rural, economically improving areas.

Susan J. Allison

Original article Zhang, L. et al. Prevalence of chronic kidney disease in China: a cross-sectional survey. Lancet 379 , 815-822 (2012)

Further reading Kovesdy, C. P. \& Kalantar-Zadeh, K. Enter the dragon: a Chinese epidemic of chronic kidney disease? Lancet 379, 783-785 (2012) 